\title{
Manejo anestésico fuera de quirófano en paciente con quemaduras en el $80 \%$ de área de superficie corporal
}

\author{
Anesthetic management outside the operating room in \\ a patient with burns $80 \%$ of the body-surface area
}

\author{
María Fernanda Alarcón Trejo, * Andrea Figueroa Martínez, ${ }^{\ddagger}$ Nora Bernal Ríos, ${ }^{\S}$ Pastor Luna Ortizף
}

Citar como: Alarcón TMF, Figueroa MA, Bernal RN, Luna OP. Manejo anestésico fuera de quirófano en paciente con quemaduras en el 80\% de área de superficie corporal. An Med ABC. 2021; 66 (3): 205-209. https://dx.doi.org/10.35366/101668

\section{RESUMEN}

En pacientes con quemaduras mayores, donde se desencadenan múltiples respuestas de citocinas proinflamatorias y destrucción masiva de tejidos, el manejo transanestésico debe cuidar cada una de las alteraciones fisiológicas con adecuado monitoreo y debe adaptarse el tratamiento del paciente fuera del área de quirófano. Se deben tener cuidados específicos como en el manejo de líquidos, la pérdida de temperatura, analgesia y el manejo de la vía aérea. Objetivo: Repaso del manejo transanestésico en paciente quemado en áreas no quirúrgicas. Reporte de caso: Presentamos el caso de un paciente masculino de 17 años de edad, quien se encuentra en el Área de Terapia Intensiva por quemaduras de tercer grado en $80 \%$ del área superficie corporal. Se programa para lavado quirúrgico y fasciotomía de miembros torácicos. Sedación con fentanilo a $2 \mu \mathrm{g} / \mathrm{kg} / \mathrm{h}$ y propofol a $1 \mathrm{mg} / \mathrm{kg} / \mathrm{h}$. Se decide realizar el procedimiento en Terapia Intensiva para evitar descompensación. Al finalizar el procedimiento se mantiene en la Unidad de Terapia Intensiva con signos vitales estables.

Palabras clave: Paciente quemado, manejo de líquidos, analgesia, monitoreo.

\begin{abstract}
In patients with major burns where multiple pro-inflammatory cytokine responses and massive destruction of tissues are triggered, transanesthetic management must take care of each of the physiological alterations with adequate monitoring and adapt the treatment of the patient outside the operating room area. Specific care should be taken, such as fluid management, loss of temperature, analgesia, and airway management. Objective: Review of transanesthetic management in burned patients in non-surgical areas. Case report: We present the case of a 17-year-old male patient who is in an intensive care area for third degree burns in $80 \%$ body total surface. It is programmed for surgical cleaning and fasciotomy of thoracic limbs. Sedation with fentanyl at $2 \mu \mathrm{g} / \mathrm{kg} / \mathrm{h}$ and propofol at $1 \mathrm{mg} /$ $\mathrm{kg} / \mathrm{h}$. It was decided to perform the procedure in intensive care to avoid decompensation. At the end of the procedure, he was kept in the Intensive Care Unit with stable vital signs.
\end{abstract}

Keywords: Burn patient, fluid management, analgesia, monitoring.

\footnotetext{
* Médico Residente de segundo año de Anestesiología.

₹ Médico Residente de tercer año de Anestesiología.

$\S$ Anestesióloga Intensivista.

ๆ Anestesiólogo Cardiovascular.
}

Centro Médico ABC. México.
Recibido: 21/04/2021. Aceptado: 17/07/2021.

Correspondencia:

Dra. María Fernanda Alarcón Trejo

E-mail: feralarcontre@gmail.com 
Abreviaturas:

ASC $=$ Área superficie corporal.

TET $=$ Tubo endotraqueal.

$\mathrm{PANI}=$ Presión arterial no invasiva

$E C G=$ Electrocardiograma.

$\mathrm{SpO}_{2}=$ Saturación parcial de oxígeno

$\mathrm{EtCO}_{2}=$ Presión parcial de $\mathrm{CO}_{2}$ al final de la espiración.

USG = Ultrasonido.

VPP = Variabilidad de presión de pulso.

PEEP = Presión positiva al final de espiración

TAM = Tensión arterial media.

UTI = Unidad de Terapia Intensiva.

$\mathrm{FC}=$ Frecuencia cardiaca

RASS = Escala de agitación y sedación de Richmond .

\section{INTRODUCCIÓN}

El manejo transanestésico en pacientes con quemaduras mayores, donde hay destrucción masiva de tejido y activación de respuesta mediada por citocinas inflamatorias, debe seguir ciertos lineamientos para lograr el mejor resultado postquirúrgico y mejorar el pronóstico a corto y largo plazo. La mortalidad sigue siendo elevada cuando los pacientes presentan ciertos factores de riesgo al momento de ser ingresados a la Unidad de Quemados.

Dentro de estos lineamientos encontramos las últimas actualizaciones, en las cuales se enfatiza el adecuado manejo de la vía aérea, monitorización y adecuada reanimación guiada por metas, considerando las lesiones coexistentes. Abordaremos de manera inicial el caso de un paciente clasificado como gran quemado y posteriormente se hará un breve resumen de lo más importante durante el perioperatorio.

\section{PRESENTACIÓN DEL CASO}

Paciente masculino de 17 años de edad sin antecedentes de importancia ni heredofamiliares según interrogatorio indirecto hacia paramédico. El paciente se encuentra en el Área de Terapia Intensiva del Centro Médico ABC con quemaduras de tercer grado en $80 \%$ del ASC clasificándose como gran quemado con 10 horas de evolución. Se programa para lavado quirúrgico y fasciotomía de miembros torácicos.

Valoración preanestésica: ASA IV-E, Detsky 15 puntos, Goldman 7 puntos. Alergias: negadas. Medicamentos: negados. Biometría hemática: leucocitos 28.9 , neutrófilos 25.9, linfocitos $2.9, \mathrm{Hg} 21.9$, plaquetas 233. Gasometría venosa: $\mathrm{pH} 7.49, \mathrm{CO}_{2} 21, \mathrm{HCO}_{3}$ 15.7, exceso de base -7, lactato 3.6. Triaje cardiaco: péptido natriurético tipo $\mathrm{B} 10$, troponina 36 , dímero D 758 , creatinina-fosfoquinasa 309 , mioglobina 65 , creatinina fosfoquinasa MB 58. Tomografía integral: importante engrosamiento cutáneo generalizado y evidente del tejido graso subcutáneo, hallazgos que pueden estar asociados a antecedentes por lesión por quemadura. Cambios que sugieren edema de la nasoy orofaringe. Quistes de retención de ambos senos maxilares. Sin evidencia de traumatismo craneoencefálico torácico y abdominal. Radiografía de columna cervical anteroposterior y lateral: rectificación de lordosis cervical. Radiografía de tórax: portátil: tráquea central, en el interior se observa tubo endotraqueal, botón aórtico y silueta cardiaca bien definidos. Hilos pulmonares de morfología y calibre normal. Pulmones distendidos. Se observa catéter venoso central de acceso derecho con extremo distal en topografía de aurícula derecha. Ultrasonido Doppler arterial de miembros torácicos: vascularidad arterial preservada en ambos miembros torácicos. Pérdida de componente diastólico de las arterias radial y cubital de forma bilateral, lo cual pudiera corresponder con restricción de la distensibilidad endotelial secundario a edema de tejidos blandos conocido. Se encuentra paciente con TET 7.5 fijo a $20 \mathrm{~cm}$ de arcada dentaria, bajo sedación con fentanilo a $2 \mu \mathrm{g} / \mathrm{kg} / \mathrm{h}$ y propofol a 1 $\mathrm{mg} / \mathrm{kg} / \mathrm{h}$. Se decide realizar procedimiento en Terapia Intensiva para evitar descompensación hemodinámica y térmica.

Monitorización: PANI, ECG, $\mathrm{SpO}_{2}, \mathrm{EtCO}_{2}$, temperatura, diuresis, se coloca línea arterial guiado por ultrasonido a nivel femoral derecho bajo técnica de asepsia y antisepsia, para monitoreo de TA, VPP y gasométrico (Figura 1).

Inducción: ketamina $70 \mathrm{mg}$, rocuronio $50 \mathrm{mg}$ y se mantiene con infusión de fentanilo a $2 \mu \mathrm{g} / \mathrm{kg} / \mathrm{h}$ y propofol a $1 \mathrm{mg} / \mathrm{kg} / \mathrm{h}$.

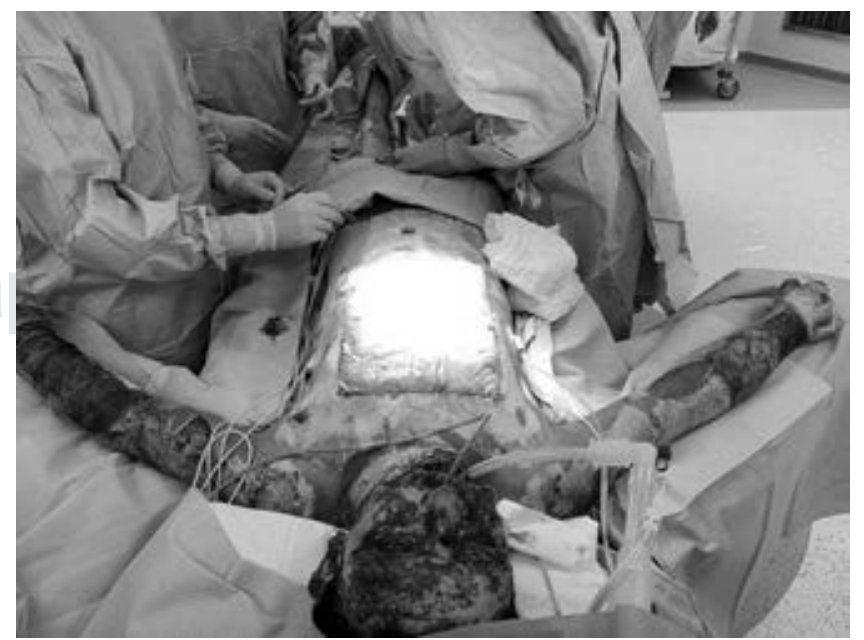

Figura 1: Paciente intubado y monitorizado adecuadamente. 
La vía aérea se mantiene bajo ventilación mecánica con medición de presión de globo del tubo endotraqueal con $23 \mathrm{cmH}_{2} \mathrm{O}$.

Mantenimiento $\mathrm{FiO}_{2} 60 \%$, volumen corriente 425 $\mathrm{mL}(6 \mathrm{~mL} / \mathrm{kg} / \mathrm{pp})$, PEEP $5 \mathrm{cmH}_{2} \mathrm{O}$, relación I:E 1.2 , Ppico $18 \mathrm{cmH}_{2} \mathrm{O}$, presión meseta de $15 \mathrm{cmH}_{2} \mathrm{O}$, resistencia de resistencia de $10 \mathrm{cmH}_{2} \mathrm{O} / \mathrm{L} / \mathrm{seg}$, distensibilidad $30 \mathrm{~mL} / \mathrm{cmH}_{2} \mathrm{O}$.

Dexmedetomidina a $0.5 \mu \mathrm{g} / \mathrm{kg} / \mathrm{h}$, se mantiene misma infusión de propofol y fentanilo.

Transanestésico: TAM $>65-70 \mathrm{mmHg}$ apoyado con norepinefrina a $0.03 \mu \mathrm{g} / \mathrm{kg} / \mathrm{min}$, tendencia a hipotermia a pesar del uso de sábana térmica, ambiente a $24{ }^{\circ} \mathrm{C}$, uso de calentador de fluidos Hotline. Aporte hídrico con solución balanceada (Hartmann + bicarbonato), sangrado de $50 \mathrm{~mL}$, diuresis de $3 \mathrm{~mL} / \mathrm{kg} / \mathrm{h}$, VPP de 9. Sin incidentes, emersión no presente. Se mantiene en la UTI con TA 100/70, FC 60 lpm, $\mathrm{SpO}_{2}$ $100 \%$, temperatura $32{ }^{\circ} \mathrm{C}$, RASS -3. Gasometría arterial al final del procedimiento $\mathrm{pH}: 7.37, \mathrm{pCO}_{2}: 46.4$, $\mathrm{HCO}_{3}: 26$, EB: $0.4, \mathrm{SO}_{2}: 97 \%$.

El paciente quemado presenta edema generalizado en quemaduras mayores del $30 \%$ de la superficie total, suele presentar pérdida de líquido intravascular en las áreas quemadas, con una pérdida de plasma continua por al menos 48 horas $^{1}$ y esto deriva en hemoconcentración. La característica del estado de choque en los pacientes quemados es el aumento de resistencia vascular sistémica debido a niveles elevados de catecolaminas, hormona antidiurética y hemoconcentración de manera inicial hasta por 48 horas, por lo que la meta inicial terapéutica es la reposición de volumen para mantener la perfusión de tejidos y minimizar la isquemia y la respuesta inflamatoria. ${ }^{2}$

En las 48 a 72 horas posteriores a la quemadura se desarrolla un estado hipermetabólico e hiperdinámico, caracterizado por el aumento del consumo de oxígeno, producción de $\mathrm{CO}_{2}$ y aumento del gasto cardiaco de 2-3 veces lo normal, taquicardia y disminución de las resistencias vasculares sistémicas. ${ }^{1}$

En la valoración inicial es crucial la identificación temprana de lesiones o rastros de quemaduras en la vía aérea, presencia de lesiones inhalatorias directas en cara o vía aérea por humo o gases, lesión química en vías superiores e inferiores, o por monóxido de carbono, datos de obstrucción o anormalidades preexistentes. ${ }^{3}$ En caso de presentarse alguna lesión es recomendable realizar una intubación temprana y no post reanimación hídrica por riesgo a desarrollar edema, lo cual amenaza la permeabilidad de la vía aérea y dificulta la ventilación o intubación. En caso de ser posible y contar con el equipo, realizar una intubación con fibroscopio en paciente despierto o a través del uso de dispositivos supraglóticos. Vigilar constantemente la disminución del edema en los tres a seis días posteriores. Determinar los factores asociados a deterioro respiratorio como daño alveolar por inhalación o lesiones torácicas que requieran escarotomías.

En caso de urgencia realizar traqueostomía para asegurar la vía aérea. Como indicación inicial aún es controvertido. Se debe administrar $\mathrm{O}_{2}$ al $100 \%$ para acortar la vida media de la carboxihemoglobina (de cuatro horas a 40-60 min) y así evitar el desplazamiento de la curva de disociación de la hemoglobina. ${ }^{3}$ Considerar la intoxicación por cianuro en pacientes con antecedente de inhalación de humo con acidosis metabólica con brecha aniónica presencia de convulsiones, insuficiencia respiratoria, coma. Presencia de $\mathrm{SvO}_{2}$ elevada-incapacidad de utilizar el $\mathrm{O}_{2}$.

Entre las medidas iniciales es primordial contar con un acceso venoso, puede ser difícil de colocar dependiendo de la extensión de las quemaduras, datos de edema o por riesgo que los cirujanos debriden el sitio de inserción del catéter. De ser necesario, utilizar venas subclavias, yugular o femoral; y en caso de ser imposible la colocación del acceso venoso, se puede colocar un catéter intraóseo. ${ }^{4}$

Fluidoterapia: evitar hipovolemia, hipoperfusión tisular, estado de choque y fallo multiorgánico. ${ }^{2}$

- Inicialmente un cristaloide isotónico y el uso de coloides 24 horas después de la lesión.

- Ringer lactato: cristaloide de elección.

- Niños: agregar dextrosa al 5\%.

- Parkland: $4 \mathrm{mg} / \mathrm{kg} / \%$ ASCQ de RL $/ 24$ horas.

- Brooke: $1.5 \mathrm{~mL} / \mathrm{kg} / \%$ ASCQ de RL/24 horas.

- ¿Inicio de coloides antes de transcurridas las primeras 24 horas? Coloide: $0.5 \mathrm{~mL} / \mathrm{kg} / \%$ ASCQ $/ 24$ horas. En caso de no responder a soluciones balanceadas se ha iniciado hasta después de ocho horas posterior a la lesión.

Es importante guiar la reposición hídrica ajustando la reanimación a metas:

1. Uresis $0.5 \mathrm{~mL} / \mathrm{kg} / \mathrm{h}$ en adultos y $1 \mathrm{~mL} / \mathrm{kg} / \mathrm{h}$ en niños.

2. TA ajustada a la normalidad por edad (presión arterial media $[\mathrm{PAM}]>65 \mathrm{mmHg}$ ).

3. FC variable (ansiedad, dolor, hipermetabolismo, catecolaminas).

4. Presión venosa central (PVC) (3-8 mmHg). 
5. Fracción de eyección de sodio $<1 \%$ (indica hipovolemia).

6. Índice BUN/creatinina $<20$.

7. ECG o USG - FEVI normal.

8. Déficit de base $\leq 5$ (hipoperfusión en ausencia de intoxicación $\mathrm{CO}$ o cianuro).

9. El del uso de concentrado eritrocitario varía en cada paciente. Plasma fresco congelado sólo en caso de hemorragia masiva, raro en pacientes quemados.

Manejo ventilatorio: volumen tidal 6-8 $\mathrm{mL} / \mathrm{kg}$, Pplat $<30 \mathrm{cmH}_{2} \mathrm{O}$. Prueba de fuga para extubación y en caso de ser posible, bajo visión directa en la sala de operaciones.

Monitoreo: $\mathrm{ECG}, \mathrm{SpO}_{2}$, PANI o línea arterial, temperatura, tren de cuatro, PVC y cuidados avanzados para traslado intrahospitalario. En el control de la temperatura es importante mantener al paciente en normotermia preferentemente, debido a que la hipotermia no es bien tolerada, ya que aumenta el consumo de $\mathrm{O}_{2}$ y exacerba la respuesta catabólica, empeora la morbilidad y mortalidad. Es necesario adoptar diferentes estrategias para mantener la temperatura adecuada (por ejemplo: sabana térmica, calentadores de soluciones, radiadores, mínima exposición, temperatura de sala de operaciones, envolver cabeza y extremidades de ser posible). ${ }^{5}$

En cuanto a los medicamentos utilizados no se recomienda la administración de antibióticos y esteroides de forma profiláctica de rutina. Existe alteración de la farmacocinética y farmacodinámica por pérdida de proteínas plasmáticas (albúmina y glicoproteína ácida $\alpha 1$ ), presentando alteración en el tiempo de acción de los medicamentos y en su eficacia. Hay un aumento del volumen de distribución de casi todos los medicamentos (propofol, fentanilo, relajantes musculares). ${ }^{6}$ Fase temprana -menor gasto cardiaco, menor flujo sanguíneo a hígado y riñones- menor eliminación. Fase hipermetabólica -mayor gasto cardiaco, más flujo a hígado y riñones- más aclaramiento.

Relajantes musculares: el uso de succinilcolina presenta riesgo de hiperkalemia, por otra parte los bloqueadores neuromusculares no despolarizantes disminuyen su sensibilidad. Posterior a las 48 horas de la lesión, las dosis necesarias pueden ser muy elevadas para lograr una adecuada relajación neuromuscular (por ejemplo: rocuronio 1.2 a $1.5 \mathrm{mg} / \mathrm{kg}$ ). ${ }^{7}$

Inductores: con base en el estado hemodinámico y la dificultad para abordar la vía aérea. El uso de halogenado no presenta influencia en la evolución del paciente; el uso de propofol requerirá dosis más altas para alcanzar hipnosis, condicionando la estabilidad hemodinámica. La ketamina es el medicamento de elección por sus propiedades analgésicas, hemodinámicamente más estable, broncodilatador y tiene efectos antiinflamatorios.

Opioides: se requieren dosis más altas, presentando tolerancia, es necesario continuar con infusiones de la UCI y aumentar en caso de ser necesario. En los pacientes con tolerancia a la morfina se pueden utilizar medicamentos adyuvantes como: clonidina, dexmedetomidina, ketamina y metadona. ${ }^{8}$

Anestesia regional: se puede utilizar como alternativas para el cultivo de injertos, tanto regionales como neuroaxiales. Precaución con catéteres cerca de sitios de quemaduras.

Nutrición parenteral: no suspender para evitar hipoglucemia.

En cuanto a la fisiopatología del dolor por quemadura existen diferentes mecanismos por los que se presenta que combinan dolor nociceptivo agudo, inflamatorio y neuropático. En el caso de nuestro paciente es un dolor agudo, por lo que la temperatura contribuye a la formación de edema y esto conlleva al inicio de la liberación en cascada de múltiples sustancias. ${ }^{6}$ Una lesión severa por quemadura lleva a una desnaturalización de proteínas y al debilitamiento de la célula plasmática por la pérdida de la integridad de la membrana, por lo tanto se liberan moléculas asociadas al patrón molecular, las cuales se unen a los receptores correspondientes.

\section{DISCUSIÓN}

El manejo del paciente quemado sigue representando un reto para el anestesiólogo, dada la magnitud del daño que estas lesiones pueden llegar a representar y las secuelas a corto y largo plazo que deben considerarse. Desde el manejo de la vía aérea hasta la farmacocinética y farmacodinamia no se debe perder de vista todo lo que implica mantener el bienestar de estos pacientes.

De acuerdo con los casos reportados en la literatura, este tipo de pacientes deben considerarse como politraumatizados para iniciar un manejo integral. Desde la evaluación adecuada de la vía aérea y el manejo correspondiente para evitar complicaciones posteriormente, hasta el manejo de líquidos y la administración de fármacos deben ser cubiertos de manera simultánea para poder garantizar una evolución adecuada.

Durante el manejo anestésico de estos casos, no debemos perder de vista que, al tratarse de pacientes 
con una farmacodinamia alterada, las dosis de nuestros fármacos pueden verse alteradas, o incluso la decisión de utilizar uno u otro inductor debe estudiarse e individualizarse de acuerdo con la fase en la que se encuentre por la lesión de quemadura.

El anestesiólogo se enfrenta a casos de pacientes quemados en diferentes fases y el manejo debe modificarse, siempre priorizando las consideraciones específicas de cada uno, ya que no será lo mismo un paciente quemado en fase temprana que otro paciente con secuelas de quemaduras.

\section{CONCLUSIONES}

Durante el manejo del paciente clasificado como gran quemado, como es el caso del paciente previamente comentado, es de vital importancia priorizar el manejo de las complicaciones que pongan en riesgo la vida del paciente de forma inmediata.

El manejo de la vía aérea debe ser abordado por el experto y considerar las dificultades que se pueden presentar.

La reanimación hídrica fue eficaz, sin exceder los objetivos ya planteados, y se individualiza en cada paciente.

El dolor durante las intervenciones dentro o fuera del quirófano puede llegar a niveles de intensidad muy elevados, por lo que puede ser de difícil control. Sin embargo, podremos dar una adecuada analgesia multimodal, conociendo que la activación periférica del nervio sensitivo después de una lesión por que- madura ocasiona tanto un dolor nociceptivo (dada por la liberación de múltiples sustancias proinflamatorias) como también dolor neuropático (por la lesión directa del nervio).

Asimismo, se realizó una adecuada monitorización del estado hemodinámico y del nivel de sedación, éstos se deben reevaluar constantemente por las variaciones que el paciente desarrolla conforme evoluciona el padecimiento.

\section{REFERENCIAS}

1. Bittner EA, Shank E, Woodson L, Martyn JA. Acute and perioperative care of the burn-injured patient. Anesthesiology. 2015; 122 (2): 448-464.

2. Guilabert P, Usúa G, Martín N, Abarca L, Barret JP, Colomina MJ. Fluid resuscitation management in patients with burns: update. Br J Anaesth. 2016; 117 (3): 284-296.

3. ISBI Practice Guidelines Committee; Steering Subcommittee; Advisory Subcommittee. ISBI practice guidelines for burn care. Burns. 2016; 42 (5): 953-1021.

4. Anderson TA, Fuzaylov G. Perioperative anesthesia management of the burn patient. Surg Clin North Am. 2014; 94 (4): 851-861.

5. Harbin KR, Norris TE. Anesthetic management of patients with major burn injury. AANA J. 2012; 80 (6): 430-439.

6. Morgan M, Deuis JR, Frosig-Jorgensen M, Lewis RJ, Cabot PJ, Gray PD et al. Burn pain: a systematic and critical review of epidemiology, pathophysiology, and treatment. Pain Med. 2018; 19 (4): 708-734.

7. Han T, Kim H, Bae J, Kim K, Martyn JA. Neuromuscular pharmacodynamics of rocuronium in patients with major burns. Anesth Analg. 2004; 99 (2): 386-392.

8. Griggs C, Goverman J, Bittner EA, Levi B. Sedation and pain management in burn patients. Clin Plast Surg. 2017; 44 (3): $535-540$. 